\title{
Assessment of Alamandine in Pulmonary Fibrosis and Respiratory Mechanics in Rodents
}

\author{
Renata Streck Fernandes $\mathbb{D}^{1},{ }^{1,2}$ Henrique Bregolin Dias $\left(\mathbb{D},{ }^{3}\right.$ Wynnie Amaral de Souza Jaques, ${ }^{1}$ \\ Tiago Becker, ${ }^{4}$ and Katya Rigatto $\mathbb{1}^{1,2}$ \\ ${ }^{1}$ Laboratório de Fisiologia Translacional, Universidade Federal de Ciências da Saúde de Porto Alegre (UFCSPA), Porto Alegre, Brazil \\ ${ }^{2}$ Programa de Pós-graduação em Ciências da Saúde, Universidade Federal de Ciências da Saúde de Porto Alegre (UFCSPA), \\ Porto Alegre, Brazil \\ ${ }^{3}$ Laboratório de Biofísica Celular e Inflamação, Pontifícia Universidade Católica do Rio Grande do Sul (PUCRS), Porto Alegre, Brazil \\ ${ }^{4}$ Departamento de Engenharia Mecânica, Universidade Federal do Rio Grande do Sul, Porto Alegre, Brazil
}

Correspondence should be addressed to Katya Rigatto; krigatto@gmail.com

Received 28 January 2021; Accepted 5 May 2021; Published 18 May 2021

Academic Editor: Peter Sever

Copyright (c) 2021 Renata Streck Fernandes et al. This is an open access article distributed under the Creative Commons Attribution License, which permits unrestricted use, distribution, and reproduction in any medium, provided the original work is properly cited.

Introduction. Pulmonary fibrosis (PF) is characterized by an accelerated decline in pulmonary function and has limited treatment options. Alamandine (ALA) is a recently described protective peptide of the renin-angiotensin system (RAS) with essential tasks in several conditions. Our group previously demonstrated that ALA is reduced by $365 \%$ in the plasma of patients with idiopathic PF, and thus, it is plausible to believe that stimulation of this peptide could represent an important therapeutic target. In this sense, this study investigates the effects of ALA in an experimental model of PF. Materials and Methods. Bleomycin (BLM) was administrated in Wistar rats, and these fibrotic animals were treated with ALA for 14 days. Body weight, histology, respiratory, and hemodynamic parameters were analyzed to study the effects of ALA. Results. ALA treatment attenuated the development of fibrosis $(P<0.0001)$, reduced respiratory system elastance $(P<0.0001)$, and preserved weight gain $(P<0.0001)$ in fibrotic animals without affecting the autonomic control of blood pressure and heart rate. Conclusion. The data from this study demonstrate the potential of ALA to alleviate pulmonary fibrosis and improve respiratory system mechanics in vivo. The promising results encourage more detailed investigations of the potential of ALA as a future and efficient antifibrotic.

\section{Introduction}

Pulmonary fibrosis (PF) is characterized by the excessive extracellular matrix (ECM) deposition and frequently evolve to death [1]. In these patients, the elastance of the respiratory system are significantly increased [2], requiring greater respiratory work. Therefore, it is essential to find effective therapeutic strategies that facilitate lung expansion, reducing episodes of respiratory failure.

The participation of renin-angiotensin system (RAS) in PF has been well described [3-5]. The angiotensin-converting enzyme 2 (ACE2) has shown been an important counterregulatory axis in several different conditions [6-8]. The alamandine (ALA), generated by ACE2 axis, although recently discovered [9], is well-known due its protective action in the cardiovascular system, i.e., vasodilation [9] and antifibrotic effects [10]. In addition, our previous study showed that patients with idiopathic PF present 365\% less ALA in plasma [11], which probably indicates that the exogenous administration of this peptide may attenuate the development of PF and, consequently, reduce the decline in lung function.

Therefore, since bleomycin (BLM) is the best characterized PF model $[12,13]$, this study is aimed at evaluating the protective effect of ALA on the development of PF in BLMinduced rats.

\section{Materials and Methods}

2.1. Animals. All procedures followed the Guide for the Care and Use of Laboratory Animals published by the US National Institute of Health [14]. Ethics Research Committee of the Universidade Federal de Ciências da Saúde de Porto Alegre 
approved the study (protocol 17/207). Five-week-old male Wistar rats were housed in a room $\left(25 \pm 2^{\circ} \mathrm{C}\right)$ on a $12: 12 \mathrm{~h}$ dark/light circadian rhythm with access to standard diet and water ad libitum.

2.2. Pulmonary Fibrosis Protocol. Rats were anesthetized with ketamine $(80 \mathrm{mg} / \mathrm{kg})$ and xylazine $(10 \mathrm{mg} / \mathrm{kg})$, and BLM $(2.5 \mathrm{mg} / \mathrm{kg}$, Bonar, Ache) or saline $(0.9 \%)$ was administered by oropharyngeal aspiration (OA). On the same day, the miniosmotic pumps (OM; Alzet 2004) containing saline or ALA (Sigma Aldrich, St. Louis, MO, USA) solution were introduced subcutaneously onto the animal's back to deliver $0.25 \mu \mathrm{l} /$ hour (ALA; $50 \mu \mathrm{g} / \mathrm{kg}$ or saline $=0.9 \%$ a day) for 14 days, respectively. The rats' health status and body weight were monitored daily.

Groups ( $N=7 /$ per group) are as follows: (1) CO: saline by OA and OM. (2) ALA: saline by OA and ALA $(50 \mu \mathrm{g} / \mathrm{kg} /$ day $)$ in the OM. (3) BLM: BLM $(2.5 \mathrm{mg} / \mathrm{kg})$ by $\mathrm{OA}$ and saline in the OM. (4) BLM+ALA: BLM $(2.5 \mathrm{mg} / \mathrm{kg})$ by $\mathrm{OA}$ and ALA $(50 \mu \mathrm{g} / \mathrm{kg} /$ day) in the OM.

2.3. Histopathology. The lung was inflated and fixed in $10 \%$ phosphate-buffered formalin, and $5 \mu \mathrm{m}$ thickness sections were stained with hematoxylin and eosin (HE) and Masson's trichrome (TM). 20 fields of each slide were examined at a magnification of 400x. The fibrosis classification was according to the modified Ashcroft score [15]. Quantitative analysis of stained collagen area was performed using the Image Pro-Plus ${ }^{\circledR} 6.0$ software (Media Cybernetics, Inc., Rockville, MD, USA).

2.4. Assessment of Respiratory Function. On day 14, animals were anesthetized with ketamine $(80 \mathrm{mg} / \mathrm{kg})$ and xylazine $(10 \mathrm{mg} / \mathrm{kg})$, positioned on a plane surgical table and tracheostomized to introduce a rigid-type cannula (2-mm ID). The cannula was fixed to the trachea by a silk thread and connected to the mechanical ventilator. Respiratory function was analyzed using a mechanical ventilator for small animals (FlexiVent, Scireq, Montréal, Canada) [16].

2.5. Hemodynamic Analyses and Autonomic Evaluation. After respiratory mechanics data collection, still under anesthesia, a polyethylene catheter (PE-50) was inserted into the right carotid to record arterial blood pressure (ABP) for 10 minutes (sample rate $=2000 \mathrm{~Hz} / \mathrm{channel}$ ). The analogical signals were digitalized by a data acquisition system (WindaqAT/CODAS, Dataq 143 Instruments Inc., OH, USA). The data were analyzed by spectral analysis to assess the sympathovagal balance in the cardiovascular system $[17,18]$. At the end of the experiment, animals were euthanized by intramuscular anasthetic overdose $(240 \mathrm{mg} / \mathrm{kg}$ of ketamine and $30 \mathrm{mg} / \mathrm{kg}$ of xylazine) for lung collection.

2.6. Statistical Analysis. The normal distribution was tested by the Shapiro-Wilk test. Parametric data analysis was performed using one-way or two-way analysis of variance (ANOVA) followed by Tukey's multiple comparison test. For data with nonnormal distribution, the Kruskal-Wallis test was used, followed by the Dunn post hoc test. The associations between data were demonstrated through Pearson's correlation test. Data analysis was performed using the GraphPad Prism 8 software and presented as mean \pm SEM. $P<0.05$ was considered statistically significant.

\section{Results}

3.1. Alamandine Attenuates Loss Weight of Pulmonary Fibrosis. From day 6, the BLM group gained less weight onwards when compared to the $\mathrm{CO}$ and ALA groups $(P<0.01)$. ALA treatment had a protective effect starting on day $8(P<0.05)$. By day 14 , PF rats gained significantly less body weight compared to the $\mathrm{CO}$ and ALA groups $(P<0.0001)$. However, animals treated with ALA maintained a similar weight to healthy animals $(P<0.0001$, vs. BLM group; Figure 1(a)).

3.2. Alamandine Attenuates the Development of Pulmonary Fibrosis. The lung parenchyma was preserved in the CO and ALA groups (score $=0$ ) compared to the BLM group $($ score $=3.6)$. ALA treatment had a potent effect (Figure 1(b)) by attenuating fibrosis $(\mathrm{BLM}+\mathrm{ALA}$ score $=1.3 ; P<0.0001 ; F=33.10)$. Moreover, collagen deposition was confined to the regions around blood vessels and airways in the CO and ALA groups. As expected, there was considerable lung interstitial collagen deposition in the BLM group $(P<0.05$ vs. CO and ALA). ALA treatment reduced significantly the collagen deposition compared to the BLM group $(P<0.001 ; F=8.285)$. Cellular and alveolar areas were similar among groups in (Figure 1(b)). Images of lung histology are shown in Figure 1(c).

3.3. Alamandine Treatment Improves the Respiratory Mechanics in Pulmonary Fibrosis. There was significant increase in the dynamic elastance (Edyn) in the BLM group $\left(7.27 \pm 1.6 \mathrm{cmH}_{2} \mathrm{O} / \mathrm{ml}\right)$ compared to the CO $\left(2.13 \pm 0.10 \mathrm{cmH}_{2} \mathrm{O} / \mathrm{ml}\right)$ and ALA $\left(1.92 \pm 0.08 \mathrm{cmH}_{2} \mathrm{O} / \mathrm{ml}\right)$ groups (Figure $2(\mathrm{a})$ ). The BLM+ALA group demonstrated a significant attenuation in Edyn $\left(2.22 \pm 0.18 \mathrm{cmH}_{2} \mathrm{O} / \mathrm{ml}\right)$ compared to the BLM group $(P<0.001 ; \quad F=11.28$; Figure 2(a)).

As expected, the dynamic compliance was lower in the $\mathrm{BLM}$ group $\left(0.18 \pm 0.04 \mathrm{cmH}_{2} \mathrm{O} / \mathrm{ml}\right)$ vs. $\mathrm{CO}$ $\left(0.48 \pm 0.02 \mathrm{cmH}_{2} \mathrm{O} / \mathrm{ml}\right)$ and ALA $\left(0.53 \pm 0.02 \mathrm{cmH}_{2} \mathrm{O} / \mathrm{ml}\right.$ ) groups. The results from the BLM+ALA $\left(0.47 \pm 0.04 \mathrm{cmH}_{2} \mathrm{O} / \mathrm{ml}\right)$ demonstrated a protective role of ALA on respiratory mechanics $(P<0.0001$ vs. BLM; $F=$ 20.50; Figure 2(b)). Moreover, the BLM group showed significantly higher respiratory dynamic resistance $(P<0.001$ vs. $\mathrm{CO} ; F=8.672$; Figure $2(\mathrm{c}))$, indicating the protective effect ALA in the BLM+ALA group $(P<0.001)$ to prevent loss of tissue function.

The correlation between the Ashcroft score and pulmonary elastance is shown in Figure 3. There was a strong positive correlation between the BLM and BLM+ALA groups $\left(r=0.8452 ; R^{2}=0.7143 ; P=0.0005 ;\right.$ Figure $\left.3(\mathrm{a})\right)$ probably due to the data from the BLM group alone $\left(r=0.9295 ; R^{2}\right.$ $=0.8640 ; P=0.0073$; Figure $3(\mathrm{~b}))$. Observing the correlation between those parameters in the BLM+ALA group, it is 


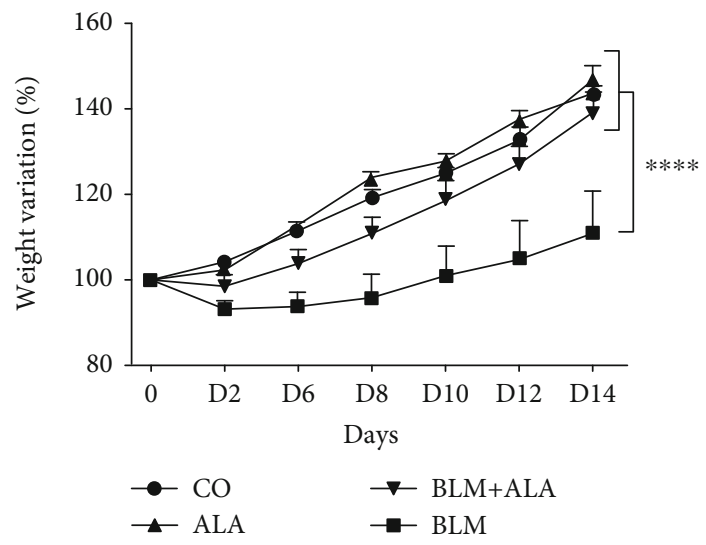

(a)
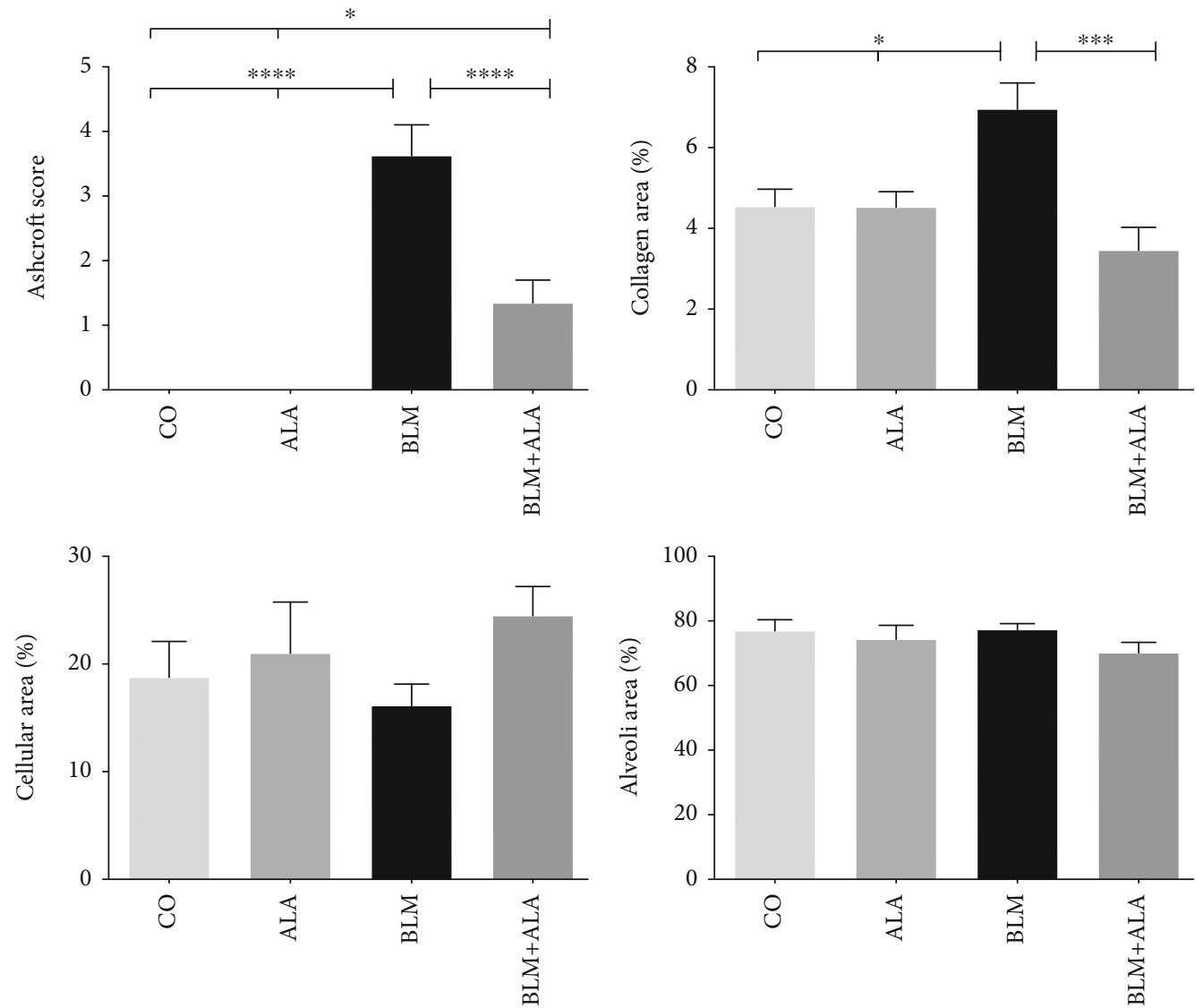

(b)

FIgure 1: Continued. 


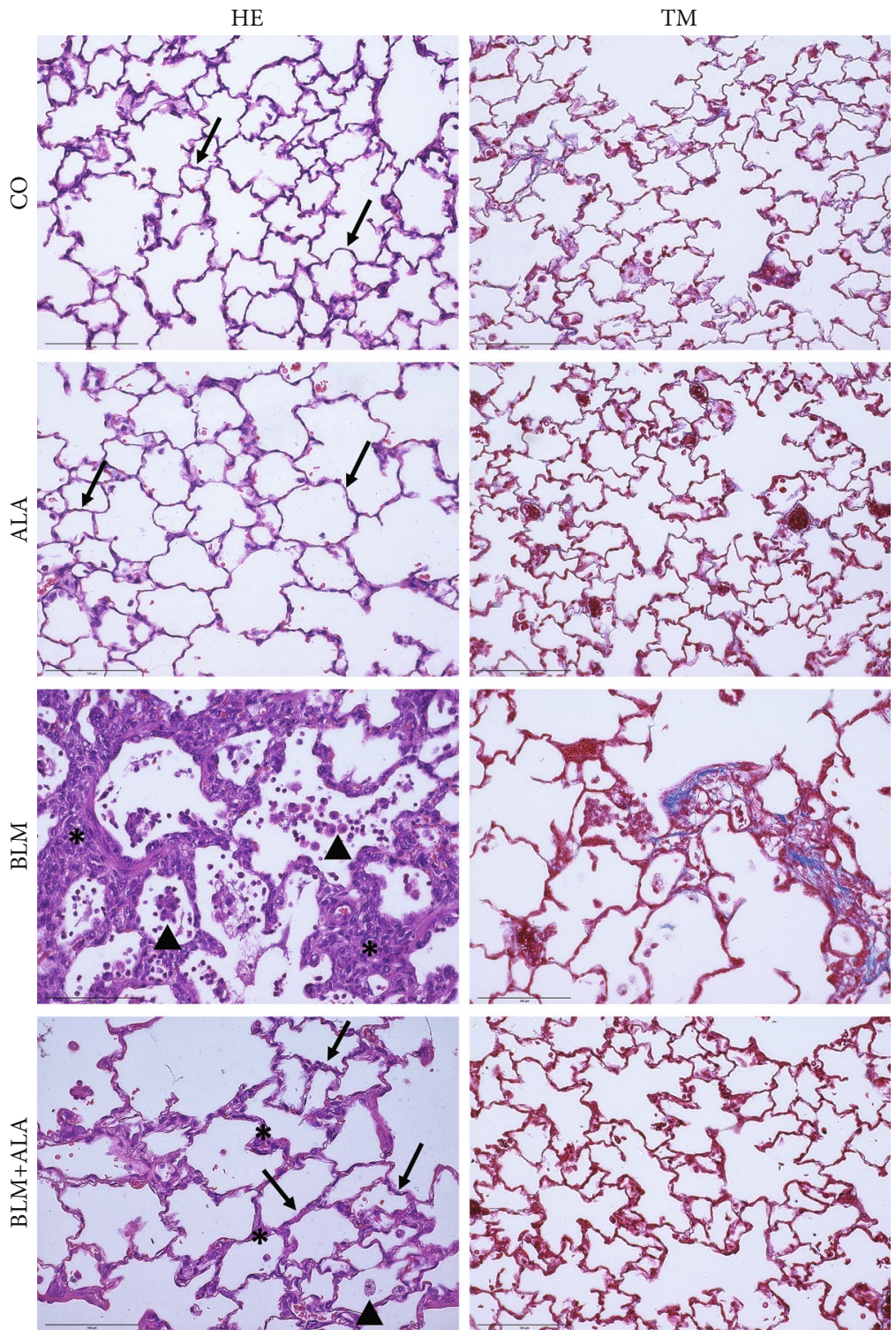

(c)

FIGURE 1: Effect of alamandine on development of pulmonary fibrosis. (a) Bodyweight variation (\%) analyzed by two-way analysis of variance (ANOVA) followed by Tukey's multiple comparison posttest. (b) Ashcroft modified score $(F=33.10)$ and collagen area quantification in the lungs $(F=8.285)$. (c) Representative images of effects at two weeks after alamandine treatment on histological findings and collagen deposition in the lungs. Hematoxylin and eosin (HE) and Masson's trichrome (TM) staining. Magnification at 400x. CO: animals that received only saline; ALA: saline intratracheally and alamandine in the osmotic minipumps; BLM: bleomycin intratracheally and saline in the osmotic minipumps; BLM+ALA: bleomycin intratracheally and alamandine in the osmotic minipumps. Arrows: alveolar septa; asterisks: fibrous bands or fibrous masses; arrowhead: inflammatory cells. Tissue changes were analyzed by one-way ANOVA, followed by Tukey's multiple comparison posttest. All data represent mean $\pm \mathrm{SEM} ; n=7-9 . P<0.05$ was considered statistically significant. ${ }^{*} P<0.05 ;{ }^{* * *} P<0.001 ;{ }^{* * * *} P<0.0001$.

possible to verify that this association was weak $(r=0.3670$; $R^{2}=0.1347 ; P=0.4742$; Figure 3(c)).

3.4. Hemodynamic Analyses. Table 1 shows that there were no differences among the hemodynamic parameters. The $\operatorname{ABP}(P=0.8121)$ and heart rate $(P=0.1432)$ were not different among groups. This result was confirmed by spectral analysis showing that the autonomic nervous system participation in the heart was also not different among groups. The sympathetic (LF) and parasympathetic components (HF) were similar in absolute and normalized units. These results were confirmed by the LF/HF ratio. In addition, the $0 \mathrm{~V}$, which represents the sympathetic participation to the heart through symbolic analysis, also indicates that there was no significant difference among groups.

\section{Discussion}

We have demonstrated for the first time the protective effect of ALA in attenuating PF and preserving respiratory mechanics. In addition, BLM administration significantly induced weight loss, which was prevented by ALA treatment. 


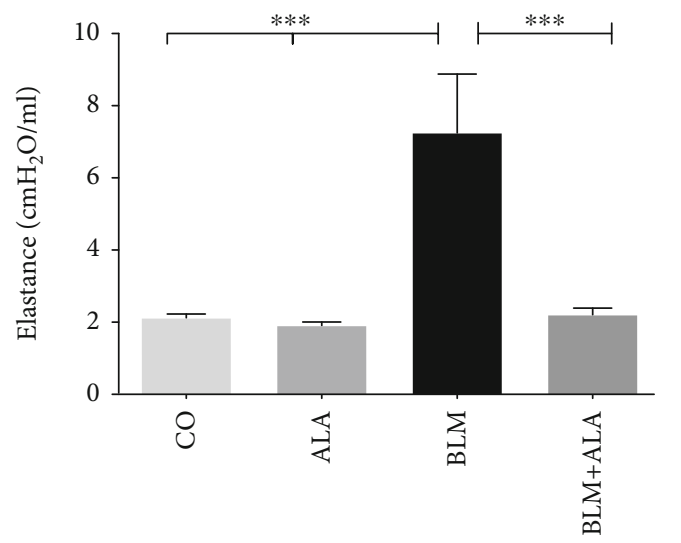

(a)

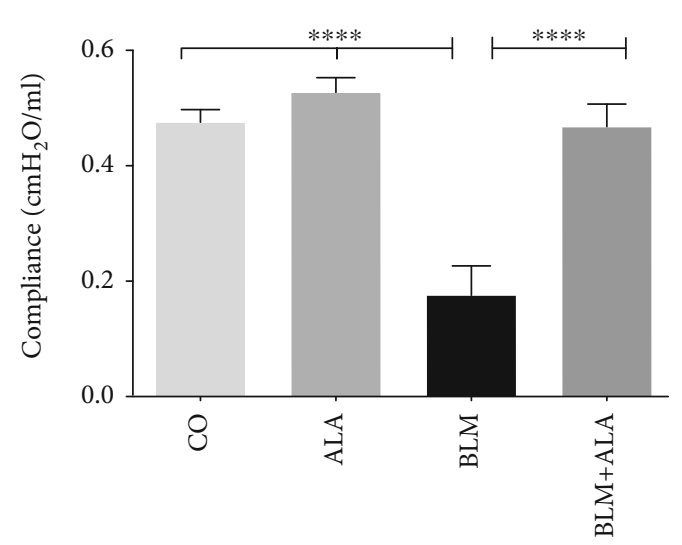

(b)

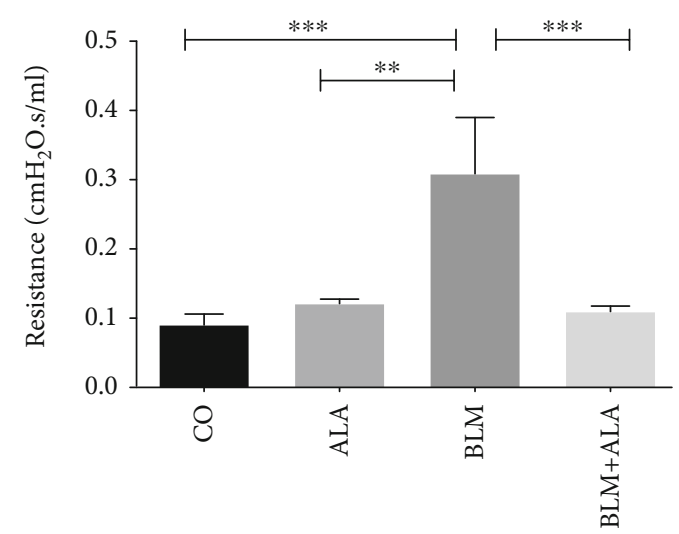

(c)

Figure 2: Lung mechanics on day 14. (a) Elastance, $F=11.28$. (b) Compliance, $F=20.50$. (c) Resistance: level of constriction, $F=8.672$. CO: animals that received only saline; ALA: saline intratracheally and alamandine in the osmotic minipumps; BLM: bleomycin intratracheally and saline in the osmotic minipumps; BLM+ALA: bleomycin intratracheally and alamandine in the osmotic minipumps. One-way ANOVA followed by Tukey's multiple comparison test was used. Data represent mean \pm SEM; $n=6-7 ; P<0.05$ was considered statistically significant. ${ }^{* *} P<0.01 ;{ }^{* * *} P<0.001 ;{ }^{* * *} P<0.0001$.

According to American Thoracic Society recommendations, induction by intratracheal BLM in male rats is the one that best mimics the disease in humans and is the most suitable for initial preclinical tests [19]. Kilic et al. [20] showed that $2.5 \mathrm{mg} / \mathrm{kg}$ of BLM in Wistar rats causes histological changes compatible with PF. Our study also demonstrated that this dose is sufficient to study the effects of antifibrotic substances without causing lethal damage to animals. In addition, George et al. [21] describes that studies for antifibrotic therapies using the BLM animal model of PF can be beneficial also to COVID-19. Even in patients recovered from COVID-19, the virus elimination does not preclude the development of progressive fibrosis. Thus, the promising results of ALA obtained in our study might encourage investigations of preventive fibrosis therapies after SARS-CoV-2 infection.

Our findings agree with the literature showing that PF leads to a decrease in body weight [22], a strong indication of health in animals [22]. While ALA treatment prevented the loss in body weight, the higher energy consumption to respiratory work probably explains this decrease in BLM group. This is true also for humans because treatment with nintedanib or pirfenidone normally provokes weight loss in patients [23], contributing to their poor prognosis [24].

Furthermore, the antifibrotic effect of ALA has already been described in the cardiovascular system [25] and, more recently, in the liver [26]. Although there are strong indications of the protective effects of ALA, there are no reports in the literature investigating its action on the fibrotic process in the lung. To date, there are only studies suggesting the protective role of to ACE2 and angiotensin-(1-7) in PF [27] and COVID-19 [8, 28]. In this study, ALA alleviated the lung degree of fibrosis and collagen deposition. It is established in the literature that collagen is the primary determinant of overall lung tissue elasticity [29], which therefore commits to functional capacity [30] in the same proportion as the degree of fibrosis [31]. Thus, if ALA prevent the fibrosis, it is possible that ALA can also act by improving respiratory mechanics.

Furthermore, our findings show that the degree of fibrosis was positively correlated with respiratory system elastance and that ALA treatment reduced this correlation. It is well 


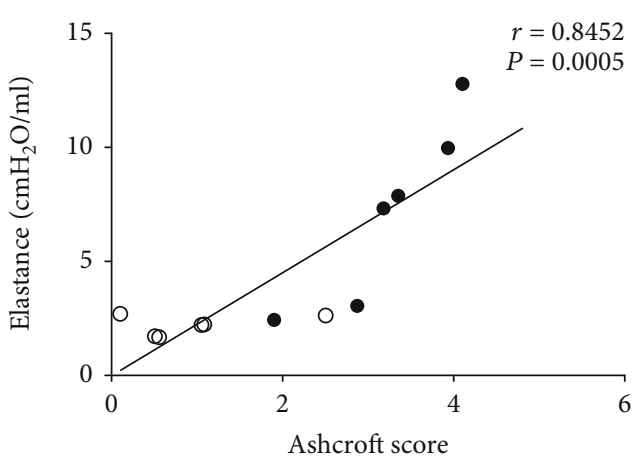

(a)

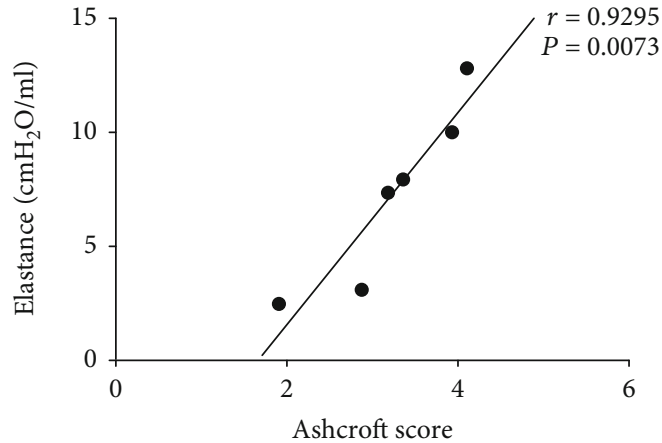

(b)

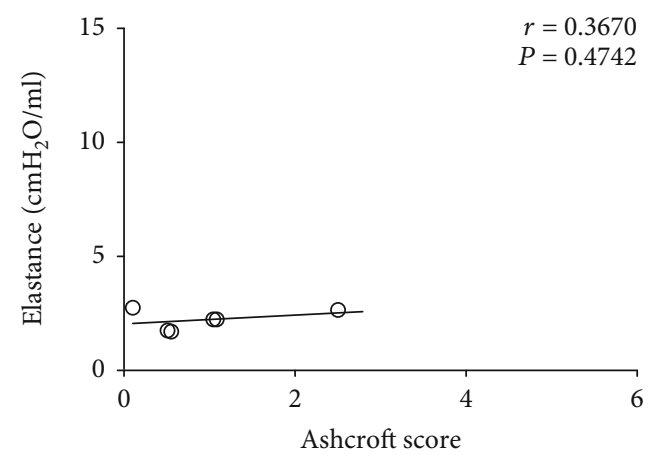

(c)

Figure 3: Pearson's correlation between Ashcroft score and pulmonary elastance. (a) BLM and BLM+ALA groups $\left(R^{2}=0.7143\right)$. (b) BLM group $\left(R^{2}=0.8640\right)$. (c) BLM+ALA group $\left(R^{2}=0.1347\right)$. BLM: bleomycin intratracheally and saline in the osmotic minipumps; BLM +ALA: bleomycin intratracheally and alamandine in the osmotic minipumps; $N=7$. $\bullet$ : BLM group. ${ }^{\circ}$ BLM+ALA group.

TABLE 1: Hemodynamic data and spectral and symbolic analysis results. CO: control rats; ALA: rats treated only with alamandine; BLM: rats treated with bleomycin; BLM+ALA: rats treated with bleomycin+alamandine. HR: heart rate; bpm: beats per minute; ABP: average blood pressure. HRV: heart rate variability; LF: low- and HF: high-frequency component; a: absolute and nu: normalized units. A one-way analysis of variance (ANOVA) followed by Tukey's multiple comparison posttest was used for ABP and HR evaluation. The KruskalWallis and the post hoc Dunn's multiple comparison tests were performed to detect differences in spectral and symbolic analysis. Data represent mean \pm SEM, and a $P<0.05$ was considered statistically significant.

\begin{tabular}{|c|c|c|c|c|c|}
\hline \multicolumn{6}{|c|}{ Spectral analysis } \\
\hline & $\mathrm{CO}(n=7)$ & $\operatorname{ALA}(n=7)$ & $\operatorname{BLM}(n=7)$ & $\mathrm{BLM}+\mathrm{ALA}(n=7)$ & $P$ \\
\hline $\mathrm{ABP}(\mathrm{mmHg})$ & $75 \pm 6$ & $70 \pm 3$ & $74 \pm 5$ & $77 \pm 3$ & 0.81 \\
\hline HR (bpm) & $299 \pm 16$ & $269 \pm 17$ & $251 \pm 11$ & $267 \pm 10$ & 0.14 \\
\hline $\operatorname{HRV}\left(\mathrm{ms}^{2}\right)$ & $9.18 \pm 2.26$ & $9.58 \pm 2.81$ & $9.43 \pm 4.28$ & $12.69 \pm 6.68$ & 0.90 \\
\hline $\mathrm{LFa}\left(\mathrm{ms}^{2}\right)$ & $2.12 \pm 0.74$ & $3.15 \pm 1.38$ & $1.31 \pm 0.37$ & $2.12 \pm 0.86$ & 0.68 \\
\hline $\mathrm{HFa}\left(\mathrm{ms}^{2}\right)$ & $5.50 \pm 1.63$ & $4.30 \pm 0.98$ & $7.07 \pm 4.12$ & $6.03 \pm 2.47$ & 0.96 \\
\hline LFnu & $0.32 \pm 0.09$ & $0.32 \pm 0.07$ & $0.28 \pm 0.08$ & $0.30 \pm 0.07$ & 0.96 \\
\hline HFnu & $0.68 \pm 0.09$ & $0.68 \pm 0.07$ & $0.72 \pm 0.08$ & $0.70 \pm 0.07$ & 0.96 \\
\hline $\mathrm{LF} / \mathrm{HF}$ ratio & $0.83 \pm 0.38$ & $0.62 \pm 0.19$ & $0.56 \pm 0.21$ & $0.50 \pm 0.16$ & 0.97 \\
\hline \multicolumn{6}{|c|}{ Symbolic analysis (\%) } \\
\hline $0 \mathrm{~V}$ pattern & $0.101 \pm 0.006$ & $0.100 \pm 0.019$ & $0.111 \pm 0.027$ & $0.111 \pm 0.010$ & 0.99 \\
\hline $1 \mathrm{~V}$ pattern & $0.370 \pm 0.010$ & $0.372 \pm 0.021$ & $0.361 \pm 0.017$ & $0.392 \pm 0.010$ & 0.40 \\
\hline 2LV pattern & $0.102 \pm 0.014$ & $0.091 \pm 0.012$ & $0.103 \pm 0.012$ & $0.075 \pm 0.014$ & 0.34 \\
\hline 2UV pattern & $0.418 \pm 0.020$ & $0.434 \pm 0.031$ & $0.424 \pm 0.035$ & $0.413 \pm 0.020$ & 0.94 \\
\hline
\end{tabular}


established that fibrosis increases the elastic recoil forces of the lung and therefore reduces lung compliance. Moreover, excess ECM alters ventilation/perfusion ratios in the lung, causing hypoxemia both at rest and with effort [32]. Consequently, our results indicate that treatment with ALA might overcome these mechanical changes and could be effective in reducing respiratory work also in IPF patients. Despite evidence in the literature regarding the involvement of the RAS in PF [33], this is the first study which demonstrates the protective effect of ALA in lungs, extending the knowledge about the potential of the ACE2 axis [34]. In this sense, our data point to the possibility of using ALA to treat PF of varying etiology, mainly when involving ACE2 participation. As proposed by $\mathrm{Wu}$ [8], our findings indicate that the compensation of ACE2 function, with ALA administration, could be a promising alternative to treat the severe respiratory damage provoked by PF, as found in COVID-19 [21, 28].

Studies have also indicated that ALA has different effects on arterial blood pressure (ABP) regulation, depending on where it is administered and/or the pathophysiological condition. It causes an increase in ABP and sympathetic participation when injected into the paraventricular nucleus of spontaneously hypertensive rats [35], whereas subcutaneous infusion of ALA attenuates hypertension [36]. It was also demonstrated by Wang et al. [37] that ALA attenuates cardiac fibrosis caused by long-term hypertension independently of ABP. Similar to the report by Wang et al. in 2019 [37], our results demonstrate that ALA attenuated lung fibrosis but did not change $\mathrm{ABP}$, clearly indicating the versatility of ALA's effects and confirming RAS pleiotropism.

\section{Conclusion}

In the future, it is possible that ALA represents an important strategy to improve IPF patient quality of life. This histological and functional study supports a significant progress and may encourage further investigation into the mechanisms of ALA in PF.

\section{Data Availability}

The experimental data that support the findings of this study are included within the article.

\section{Conflicts of Interest}

All authors declare no conflict of interest.

\section{Acknowledgments}

This work was conducted during a PhD Scholarship at the University financed by Coordenação de Aperfeiçoamento de Pessoal de Nível Superior (CAPES) (grant number 88882.442836/2019-01). We thank the Pathology Laboratory and Experimental Physiology Laboratory of UFCSPA for their contributions.

\section{References}

[1] G. Raghu, B. Rochwerg, Y. Zhang et al., "An official ATS/ERS/JRS/ALAT clinical practice guideline: treatment of idiopathic pulmonary fibrosis: an update of the 2011 clinical practice guideline," American Journal of Respiratory and Critical Care Medicine, vol. 192, no. 2, pp. e3-e19, 2015.

[2] S. Nava and F. Rubini, "Lung and chest wall mechanics in ventilated patients with end stage idiopathic pulmonary fibrosis," Thorax, vol. 54, no. 5, pp. 390-395, 1999.

[3] B. D. Uhal, X. Li, C. C. Piasecki, and M. Molina-Molina, "Angiotensin signalling in pulmonary fibrosis," The International Journal of Biochemistry \& Cell Biology, vol. 44, no. 3, pp. 465-468, 2013.

[4] E. Montes, V. Ruiz, M. Checa et al., "Renin is an angiotensinindependent profibrotic mediator: role in pulmonary fibrosis," The European Respiratory Journal, vol. 39, no. 1, pp. 141-148, 2012.

[5] M. Kreuter, D. J. Lederer, M. Molina-Molina et al., "Association of angiotensin modulators with the course of idiopathic pulmonary fibrosis," Chest, vol. 156, no. 4, pp. 706-714, 2019.

[6] J. Chen, L. Zhao, D. Wang et al., "Contribution of regulatory T cells to immune tolerance and association of microRNA-210 and Foxp3 in preeclampsia," Molecular Medicine Reports, vol. 19, no. 2, pp. 1150-1158, 2019.

[7] A. Rathinasabapathy, A. J. Bryant, T. Suzuki et al., "rhACE2 therapy modifies bleomycin-induced pulmonary hypertension via rescue of vascular remodeling," Frontiers in Physiology, vol. 9, no. 271, pp. 1-10, 2018.

[8] Y. Wu, "Compensation of ACE2 function for possible clinical management of 2019-nCoV-induced acute lung injury," Virologica Sinica, vol. 35, no. 3, pp. 256-258, 2020.

[9] R. Q. Lautner, D. C. Villela, R. A. Fraga-Silva et al., "Discovery and characterization of alamandine: a novel component of the renin-angiotensin system," Circulation Research, vol. 112, no. 8, pp. 1104-1111, 2013.

[10] F. P. de Souza-Neto, M. de Morais Silva, M. de Carvalho Santuchi et al., "Alamandine attenuates arterial remodelling induced by transverse aortic constriction in mice," Clinical Science, vol. 133, no. 5, pp. 629-643, 2019.

[11] T. S. Sipriani, R. A. S. dos Santos, and K. Rigatto, "The renin-angiotensin system: alamandine is reduced in patients with idiopathic pulmonary fibrosis," Journal of Cardiology and Cardiovascular Medicine, vol. 4, no. 3, pp. 210-215, 2019.

[12] R. Carrington, S. Jordan, S. C. Pitchford, and C. P. Page, "Use of animal models in IPF research," Pulmonary Pharmacology \& Therapeutics, vol. 51, pp. 73-78, 2018.

[13] I. Barbayianni, I. Ninou, A. Tzouvelekis, and V. Aidinis, "Bleomycin revisited: a direct comparison of the Intratracheal micro-spraying and the oropharyngeal aspiration routes of bleomycin administration in mice," Frontiers in Medicine, vol. 5, no. 269, pp. 1-6, 2018.

[14] National Research Council, Guide for the Care and Use of Laboratory Animals, E. Edi, Ed., The National Academies Press, DC, 2011.

[15] R.-H. Hübner, W. Gitter, N. E. El Mokhtari et al., "Standardized quantification of pulmonary fibrosis in histological samples," BioTechniques, vol. 44, no. 4, pp. 507-517, 2008.

[16] F. Rago, E. M. Melo, L. Kraemer et al., "Effect of preventive or therapeutic treatment with angiotensin 1-7 in a model of 
bleomycin-induced lung fibrosis in mice," Journal of Leukocyte Biology, vol. 106, no. 3, pp. 677-686, 2019.

[17] K. Rigatto, K. R. Casali, V. Shenoy, M. J. Katovich, and M. K. Raizada, "Diminazene aceturate improves autonomic modulation in pulmonary hypertension," European Journal of Pharmacology, vol. 713, no. 1-3, pp. 89-93, 2013.

[18] E. Quagliotto, K. R. Casali, P. Dal Lago, and A. A. Rasia-Filho, "Neuropeptides in the posterodorsal medial amygdala modulate central cardiovascular reflex responses in awake male rats," Brazilian Journal of Medical and Biological Research, vol. 48, no. 2, pp. 128-139, 2015.

[19] R. G. Jenkins, B. B. Moore, R. C. Chambers et al., "An official American thoracic society workshop report: use of animal models for the preclinical assessment of potential therapies for pulmonary fibrosis," American Journal of Respiratory Cell and Molecular Biology, vol. 56, no. 5, pp. 667-679, 2017.

[20] T. Kilic, H. Parlakpinar, A. Polat et al., "Protective and therapeutic effect of Molsidomine on bleomycin-induced lung fibrosis in rats," Inflammation, vol. 37, no. 4, pp. 1167-1178, 2014.

[21] P. M. George, A. U. Wells, and R. G. Jenkins, "Pulmonary fibrosis and COVID-19: the potential role for antifibrotic therapy," The Lancet Respiratory Medicine, vol. 8, no. 8, pp. 807$815,2020$.

[22] J. Wang, C. Fang, S. Wang et al., "Danggui Buxue tang ameliorates bleomycin-induced pulmonary fibrosis in rats through inhibiting transforming growth factor- $\beta 1 / \mathrm{Smad} 3 /$ plasminogen activator inhibitor-1 signaling pathway," Journal of Traditional Chinese Medicine $=$ Chung $i$ Tsa Chih Ying Wen Pan, vol. 40, no. 2, pp. 236-244, 2020.

[23] A. Perelas, J. Glennie, K. van Kerkhove et al., "Choice of antifibrotic medication and disease severity predict weight loss in idiopathic pulmonary fibrosis," Pulmonary Pharmacology \& Therapeutics, vol. 59, article 101839, 2019.

[24] Y. Nakatsuka, T. Handa, M. Kokosi et al., "The clinical significance of body weight loss in idiopathic pulmonary fibrosis patients," Respiration, vol. 96, no. 4, pp. 338-347, 2018.

[25] M. de Carvalho Santuchi, M. F. Dutra, J. P. Vago et al., "Angiotensin-(1-7) and alamandine promote anti-inflammatory response in macrophages in vitro and in vivo," Mediators of Inflammation, vol. 2019, Article ID 2401081, 14 pages, 2019.

[26] Y. Huang, Y. Li, A. Lou et al., "Alamandine attenuates hepatic fibrosis by regulating autophagy induced by NOX4-dependent ROS," Clinical Science, vol. 134, no. 7, pp. 853-869, 2020.

[27] L. Wang, Y. Wang, T. Yang, Y. Guo, and T. Sun, “Angiotensinconverting enzyme 2 attenuates bleomycin-induced lung fibrosis in mice," Cellular Physiology and Biochemistry, vol. 36, no. 2, pp. 697-711, 2015.

[28] C. Annweiler, Z. Cao, W. Yingliang et al., "Counter-regulatory 'renin-angiotensin' system-based candidate drugs to treat COVID-19 diseases in SARS-CoV-2-infected patients," Infectious Disorders - Drug Targets, vol. 20, pp. 19-20, 2020.

[29] B. Suki and J. H. T. Bates, "Lung tissue mechanics as an emergent phenomenon," Journal of Applied Physiology, vol. 110, no. 4, pp. 1111-1118, 2011.

[30] R. M. du Bois, D. Weycker, C. Albera et al., "Forced vital capacity in patients with idiopathic pulmonary fibrosis: test properties and minimal clinically important difference," American Journal of Respiratory and Critical Care Medicine, vol. 184, no. 12, pp. 1382-1389, 2011.
[31] K. L. Olukogbon, P. Thomas, R. Colasanti, B. Hope-Gill, and E. M. Williams, "Breathing pattern and breathlessness in idiopathic pulmonary fibrosis: an observational study," Respirology, vol. 21, no. 2, pp. 344-349, 2016.

[32] D. E. Amariei, N. Dodia, J. Deepak et al., "Combined pulmonary fibrosis and emphysema: pulmonary function testing and a pathophysiology perspective," Medicina, vol. 55, no. 9, p. 580, 2019.

[33] X. Yu, Y. Zhang, X. Yang et al., "The influence of BuqiHuoxueTongluo formula on histopathology and pulmonary function test in bleomycin-induced idiopathic pulmonary fibrosis in rats," Evidence-Based Complementary and Alternative Medicine, vol. 2018, Article ID 8903021, 13 pages, 2018.

[34] F. Zhou, T. Yu, R. Du et al., "Clinical course and risk factors for mortality of adult inpatients with COVID-19 in Wuhan, China: a retrospective cohort study," The Lancet, vol. 395, no. 10229, pp. 1054-1062, 2020.

[35] Y. H. Shen, X. R. Chen, C. X. Yang, B. X. Liu, and P. Li, “Alamandine injected into the paraventricular nucleus increases blood pressure and sympathetic activation in spontaneously hypertensive rats," Peptides, vol. 103, pp. 98-102, 2018.

[36] R. N. Vuille-dit-Bille, S. M. Camargo, L. Emmenegger et al., "Human intestine luminal ACE2 and amino acid transporter expression increased by ACE-inhibitors," Amino Acids, vol. 47, no. 4, pp. 693-705, 2015.

[37] L. Wang, C. Liu, X. Chen, and P. Li, “Alamandine attenuates long-term hypertension-induced cardiac fibrosis independent of blood pressure," Molecular Medicine Reports, vol. 19, no. 6, pp. 4553-4560, 2019. 